G. Meylan

University of California, Berkeley

\begin{abstract}
The southern sky gives us the great opportunity to observe two among the brightest and nearest globular clusters of the Galaxy: $\omega$ Cen and 47 Tuc . For these giant clusters, we present the comparison between observations and KingMichie multi-mass dynamical models with anisotropy in the velocity dispersion. A more comprehensive description of this work is to be published (Meylan 1986a,b).
\end{abstract}

\title{
1. THE OBSERVATIONS
}

The present dynamical description uses both surface brightness and velocity dispersion profiles. Precise radial velocities have been obtained with the photoelectric spectrometer CORAVEL at the European Southern Observatory at Cerro La Silla, Chile, in collaboration with astronomers in Geneva, Marseilles, Copenhagen, and ESO (Mayor et al. 1983, 1986). The number of observations amounts in $\omega$ Cen to 540 measurements of 318 member stars, and in 47 Tuc to 371 measurements of 272 member stars, with typical uncertainties of 0.9 and $0.6 \mathrm{~km} / \mathrm{s}$, respectively.

\section{THE MODEL}

Models have been constructed in an approach nearly identical to Gunn and Griffin (1979). Heavy remnants (e.g. neutron stars), white dwarfs, and MS stars have been distributed into ten different subpopulations, each obeying the energyangular momentum distribution function: $f_{i}(E, J) \propto\left[\exp \left(-A_{i} E\right)-1\right] \exp \left(-\beta J^{2}\right)$. In the cluster center, thermal equilibrium is assumed in order to force $A_{i}$ to be proportional to the mean mass of the stars in the subpopulation considered. A model is specified by a mass function exponent $x$, and by four parameters: the scale radius $r_{c}$, the scale velocity $v_{s}$, the central value of the potential $W_{\circ}$, and the anisotropy radius $r_{a}$. Beyond $r_{a}$, the velocity dispersion tensor is mostly radial.

\section{THE RESULTS}

Models have been calculated for a wide range of values of each parameter.

For $\omega$ Cen, the observations are well fitted only by models with strong anisotropy, with $r_{a} \simeq 2-3 r_{c}$ (Fig. 1). This is related to the large half-mass relaxation time $t_{r h} \simeq 20-3010^{9} \mathrm{yr}\left(t_{r}(0) \simeq 10^{9} \mathrm{yr}\right)$. The mean value of the exponent $\mathrm{x}$ of the mass 663 
function is $\simeq 1.25$, close to Salpeter's 1.35 . The heavy remnants represent from 0 to $9 \%$ of the total mass, being anticorrelated with the white dwarfs. The mean total mass of the cluster is about $410^{6} M_{\odot}$, giving a mean $M / L_{V} \simeq 3$.

In 47 Tuc, using the flat luminosity function of Harris and Hesser $(1985 \mathrm{HH})$, the best models (Fig. 2) have an anisotropy radius $r_{a} \simeq 20-40 r_{c}$. The high concentration involves $t_{r h} \simeq 310^{9} \mathrm{yr}\left(t_{r}(0) \simeq 10^{7} \mathrm{yr}\right)$. Heavy remnants are not needed, in contrast to white dwarfs which represent always about $30 \%$ of the mass. The mean total mass is about $0.710^{6} M_{\odot}$, giving a mean $M / L_{V} \simeq 1.8$.

\section{REFERENCES}

Mayor, M., Imbert, M., Anderson, J., Ardeberg, A., Baranne, A., Benz, W., Ishci, E., Lindgren, H., Martin, N., Maurice, E., Meylan, G., Nordström, B. and Prévot, L. 1983, 1986 Astron. Astrophys.

Supp1. 54, 495 and in press.

Meylan, G. 1968a,b, in preparation.
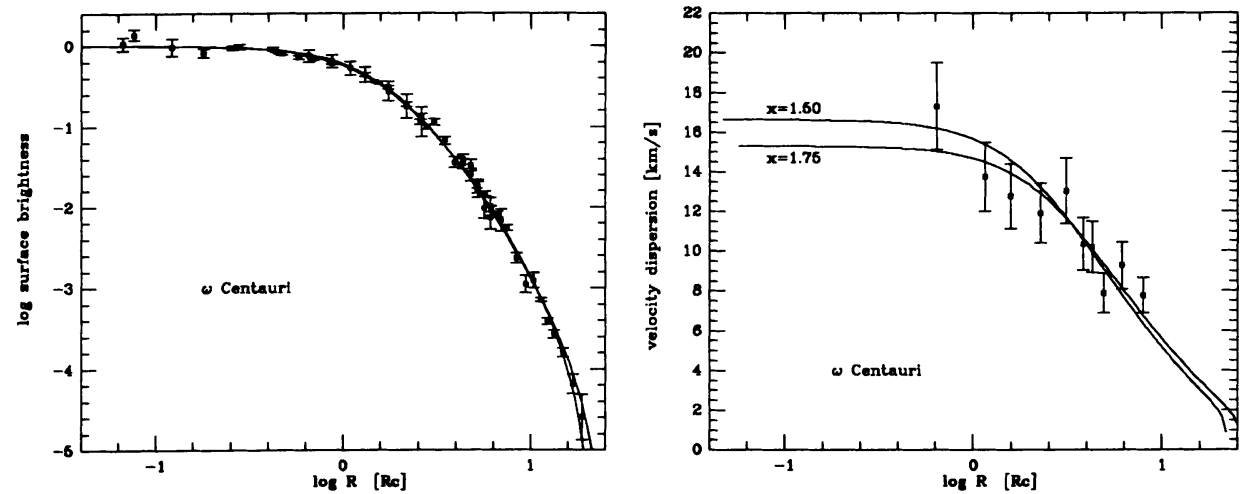

Fig. $1 \omega$ Cen: observed and computed surface brightness and velocity dispersion profiles, for the models with $m_{h r}=2.0, x=1.50$ and $1.75, W_{0}=8.5$, and $r_{a}=3.0$.
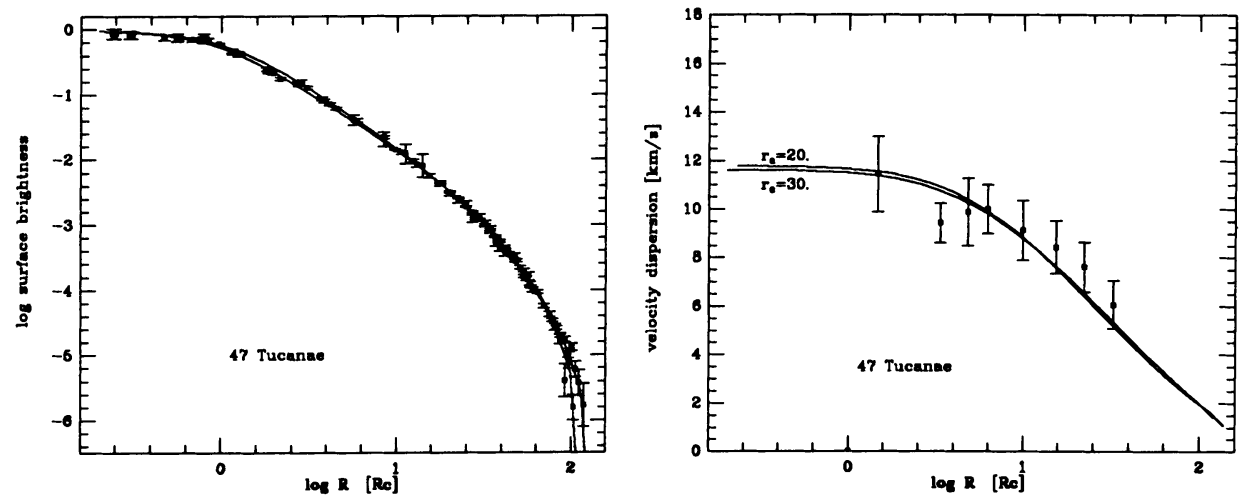

Fig. 247 Tuc: observed and computed surface bright. and velocity disp. profiles, for the models with $m_{h r}=1.4, x=H H, W_{\circ}=10$. and 9.5, and $r_{a}=30$. and 20 . 